\title{
Material Properties \\ Determination of Anisotropic Mechanical Properties of G-10 Composite via Direct Strain Imaging
}

\author{
Athanasios Iliopoulos ${ }^{\mathrm{a}}$, John Steuben ${ }^{\mathrm{b}}$ John Michopoulos ${ }^{\mathrm{b} \dagger}$ \\ ${ }^{a}$ George Mason University, \\ resident at Computational Multiphysics Systems Lab, Code 6394, \\ Naval Research Laboratory, Washington, DC 20375 USA \\ ${ }^{\mathrm{b}}$ Computational Multiphysics Systems Lab, Code 6394, \\ Naval Research Laboratory, Washington, DC 20375 USA \\ †Please address all correspondence to: \\ John Michopoulos, john.michopoulos@nrl.navy.mil
}

\begin{abstract}
The mechanical properties of the glass-epoxy composite material known as "G-10," which is primarily used for electrical insulation purposes in electromechanical systems, have long been a topic of uncertainty and controversy. In the present paper, we demonstrate the application of an experimental methodology that exploits the Direct Strain Imaging (DSI) full field method to fully assess the elastic anisotropic mechanical properties of G-10. Tension and compression experiments were conducted for three distinct inclinations of the specimen orthotropic axes relative to the loading directions, while strain and displacement were monitored at relevant locations on the specimens via digital image capturing and subsequent application of DSI. Based on these experiments, we present the stress-strain experimental data, the associated data post processing, the engineering properties, the anisotropic compliance matrix and the ultimate strength properties of the G-10 composite material. Confidence bounds on the material properties based on the results from repeated tests on identical specimens are also reported.
\end{abstract}

Keywords: G-10 Composite, FR4, Direct Strain Imaging, Full-field Methods, Anisotropic Properties, Young's Modulus, Poisson ratio, Shear Modulus, Strength.

\section{Introduction}

The composite material known as "G-10" is widely used in many electromechanical applications, primarily as an electrical insulator with mechanical properties that make the material practicably useful. More precisely, G-10 is a specification for a grade of glass-fiber reinforced epoxy or "fiberglass" material, which is defined in [1-3]. This material consists of lamina of continuous glass woven roving which is impregnated with an epoxy resin binder under high pressure. G-10 is closely related to other materials such as FR4, which is a brominated fireretardant variety of G-10, and G-10 CR, which uses a non-brominated bisphenol-A epoxy resin as a matrix that makes it a cryogenic grade material. These materials are used extensively in the electronics industry because of their high electrical insulation properties, extremely low water absorption, relatively high mechanical stiffness/strength and high dimensional stability [4]. In 
the present work we used the basic G-10 variant.

In many cases, G-10 composites are used in applications where precise knowledge of the mechanical properties of the material is unimportant. However, many emerging applications for this material are based on its excellent stiffness and ultimate strength. Such applications include the construction of high-power supply systems, high-power magnetic coils [5, 6], cryogenic superconducting magnets [7] and electromagnetic launchers [8,9]. Despite this widespread and diverse variety of uses of G-10, there is very scarce information in the bibliography regarding its mechanical properties. Readily available data from manufacturers is typically limited to tensile strength and elastic modulus (ASTM D638), Flexural strength and modulus (ASTM D790) and compressive strength (ASTM D695). While these data are adequate for some design and analysis purposes, they are not sufficient to support rigorous analysis in a computational environment. In such contexts, for instance a finite-element analysis (FEA), all non-zero elements of the anisotropic compliance/stiffness matrix must be known.

Arguably, the most complete reference reporting some of the mechanical properties of the G-10 woven roving laminate is [10]. This study presents results from tension and compression experiments and provide values for the Young's modulus in the three principal directions. Unfortunately, this report does not include shear moduli or Poisson ratio data for any direction. In addition, the compression tests were not performed utilizing any elongation or strain measuring device; instead grip separation was used, which can lead to unreliable data. Additional studies of the properties of cryogenic grades of G10, commonly known as G-10CR, are given by $[11,12]$, but suffer from similar limitations. Studies of the FR4 variant of G-10 have also been conducted, such as the work of [13], but again only some of the relevant mechanical properties are identified. Finally, some recent research has been conducted into the high-velocity impact properties of materials including G-10 [14], but useful properties were not extracted from these test results.

In the present research, we applied a custom-developed Direct Strain Imaging (DSI) [1528] methodology to both tensile and compressive tests. The tests were conducted on G-10 specimens manufactured for various inclinations of the orthotropic axes relative to the loading direction. Section 2 contains the description of the experimental setup. Section 3 contains a discussion and quantitative analysis of sources of error in this experimental regimen. In Section 4, we present the methodology by which the properties of the G-10 material were determined based on experimental results. In Section 5, the experimental results are presented, in the form of the resultant engineering properties and compliance matrix for G-10. The paper closes in Section 6 with conclusions and general discussion of these results.

\section{Experimental Procedure}

The purpose of the experimental procedure is to acquire the necessary data that will enable the determination of the elastic properties of the material in terms of the set of the engineering properties $\left\{E_{1}, E_{2}, E_{3}, v_{12}, v_{13}, v_{23}, G_{12}, G_{23}, G_{13}\right\}$ where $E_{i}, v_{i j}, G_{i j}$ are the Young's moduli, Poisson ratios and shear moduli, respectively, for $i, j=1,2,3$. An alternative, equivalent and more formal representation of the elastic properties of material is given by the elements of the $4^{\text {th }}$ order compliance tensor $S$ or its inverse known as the Hooke's or elasticity or stiffness tensor $\boldsymbol{C}$ where $\boldsymbol{S}=\boldsymbol{C}^{-1}$. These two symmetric tensors can also be represented as $6 \times 6$ matrices with elements $S_{i j}$ and $C_{i j}$ respectively for $i, j=1, . ., 6$.

A schematic depiction of the experimental setup is presented in Fig. 1. The test machine 
used was a $500 \mathrm{kN}$ capacity Instron 1332 servohydraulic load frame with a digital controller. Tension specimens were gripped with $100 \mathrm{kN}$ capacity side entry hydraulic grips. Compression tests employed platens affixed to the hydraulic grips such that the lower platen was mounted on top of the lower grip and the upper one was mounted on the bottom of the upper grip.

$<$ Figure 1 placed near here $>$

The strains developed in the test specimens during loading were measured using the DSI 2D full-field measurement technique. Such techniques are much more convenient than strain gauges or extensometers because of the vastly reduced system complexity and trivial specimen preparation methods. The DSI methodology operates by tracking the kinematic evolution of centroids of marks painted on the surface of each specimen [15-28] as a function of loading increments or frames. The strain calculation is performed directly on the strain tensor, as described in [25], and provides all three components of strain (horizontal, vertical and shear). The camera used for the full-field measurement of strain was placed on a rigid bracket that is attached to one of the fixed and non-deforming columns of the loading frame. This arrangement has the advantage that it greatly reduces the relative motion of the camera with respect to the specimen, and hence significantly improves the accuracy of the $2 \mathrm{D}$ strain measurement setup. A view showing the system setup is shown in Fig. 2.

$<$ Figure 2 placed near here $>$

The video stream from the camera was processed in real-time at approximately 5 frames per second. The camera used for the acquisition was a Point-Grey Flea 3 FL3-U3-88S2C-C 8.8 megapixel camera. The lens used has a focal length of $50 \mathrm{~mm}$ with a maximum aperture of $f / 2.8$, while the tests were performed with an aperture of approximately $f / 4.0$.

The custom developed machine vision system processing involves the identification of centroids of marks painted on the surface of each specimen as well as the tracking of these centroids over time [24]. The load signal from the test frame was digitized by a USB based ADC at $100 \mathrm{~Hz}$. The increased ADC rate was used as the means to reduce the latency between the processed image data and the load cell data. The load data were combined with the tracked dot location data and, subsequently, stored in the computer for further processing.

Test specimens were constructed from sheet stock G-10 of $12.7 \mathrm{~mm}(0.5 \mathrm{in})$ thickness, purchased from a commercial supplier. A waterjet cutting machine was used to cut both tensile and compressive specimens from the sheet stock. In order to realize specimens with orthotropic axes oriented in various angles relative to the uniaxial load direction, specimens were cut from the sheet stock in several orientations. The tension specimen geometry was in accordance with ASTM D638-10 and is presented in Fig. 3. The geometry of the compression specimens is also presented in Fig. 3. After test specimens were cut from the sheet stock, they were painted on one side with white enamel spray-paint. After drying of the paint, the markers necessary for DSI were placed on the painted surface using an indelible marker. For the specific experiments, DSI was used to calculate the strain at the center point of the face of the specimen as shown by the cross mark in Fig 3.

$<$ Figure $3 \mathrm{a}$ and $3 \mathrm{~b}$ placed near here, preferably side-by-side $>$ 


\section{Out of Plane Systematic Error Analysis}

During a uniaxial loading experiment, it is expected that a small out of plane displacement will be induced because of the Poisson effect. This behavior introduces a systematic error in a 2D full field strain measurement system. Although this error can be accounted for quantitatively, in the present work we will only demonstrate that its magnitude is negligible and, therefore, may be ignored.

A depiction of the out of plane deformation and the associated projection aberration is shown in Fig. 4 for the case of a pinhole camera model. In a 2D experimental setup such as the one used in here, there is no practical way to measure the distance from the camera at every time instance. Therefore, we assume that this distance is unknown. During tensile or compressive loading, the out of plane movement of a point that would be considered (assuming no throughthickness Poisson effect) in the deformed state to be located at $\boldsymbol{x}$ and projected on $\boldsymbol{x}^{\prime}$ on the image plane, should be in fact be considered (assuming through-thickness Poisson effect) to be on $\boldsymbol{y}$ that is projected on the point at vector location $\boldsymbol{y}^{\prime}$ on the image plane.

$<$ Figure 4 placed near here $>$

In a full field measurement system and for the case of tensile loading, this effect will manifest as a reduction in both the horizontal and vertical strain components. Likewise, in the case of compressive loading, this effect will manifest as an increase in both the horizontal and vertical strain components.

The magnitude of this effect can be easily calculated by basic trigonometry of the quantities in Fig. 4. The vectors shown in Fig. 4 can be written as: $\boldsymbol{x}=\{d+s, x\}^{T}$, $\boldsymbol{x}^{\prime}=\left\{d+s+p, x^{\prime}\right\}^{T}, \boldsymbol{y}=\{d, y\}^{T}$ and $\boldsymbol{y}^{\prime}=\left\{d, y^{\prime}\right\}^{T}$, where $x, y, x^{\prime}, y^{\prime}$ are the vertical distances of the respective points from the principal axis. Without loss of generality, we can assume that $x=y$. The out of plane displacement $p$ due to the Poisson effect can be calculated based on the system of equations

$$
\left.\begin{array}{l}
v_{y z}=-\frac{\varepsilon_{z z}}{\varepsilon_{y y}} \\
\varepsilon_{z z}=\frac{t-2 p}{t}-1
\end{array}\right\},
$$

where $t$ is the thickness of the specimen. In Eqs. (1), $p$ is multiplied by 2 to indicate that the Poisson effect is distributed symmetrically through the thickness. Solving the system of Eqs. (1) with respect of $p$ yields:

$$
p=\frac{1}{2} \varepsilon_{y y} v_{y z} t
$$

The distance between the image points at locations described by the vectors $\boldsymbol{x}^{\prime}$ and $\boldsymbol{y}^{\prime}$ can be calculated by simple trigonometry leading to: 


$$
x^{\prime}-y^{\prime}=\frac{\varepsilon_{y y} v_{y z} t y d}{(d+s)(2 d+s)+\varepsilon_{y y} v_{y z} t}
$$

In the present application, we estimated the distance of the camera center to the image plane to be $d=19 \mathrm{~mm}$ and measured the distance from the image plane to the surface of the specimen at $s=300 \mathrm{~mm}$. Assuming the worst-case scenario for a strain measurement at distance $y=10 \mathrm{~mm}$, a specimen thickness of $3.00 \mathrm{~mm}$ and a Poisson ratio of $v_{y z}=0.35$ and for strain level of $1 \%=0.01$ we compute a value for this error of:

$$
x^{\prime}-y^{\prime}=10.06 \times 10^{-6} \mathrm{~mm}
$$

For a typical CMOS sensor $x^{\prime} \approx 1.0 \mathrm{~mm}$. Hence, a strain of $1 \%$ would be manifested by a displacement of $0.01 \mathrm{~mm}$. Thus, the percentage error of the out of plane deformation at this strain level is expected to be:

$$
e=\left(x^{\prime}-y^{\prime}\right) / 0.01 \mathrm{~mm}=0.10 \%
$$

On the lateral direction and due to the Poisson effect, this error is expected to be approximately 3-4 times larger because of the difference of compliance in the orthogonal directions. Hence, we anticipate that the out of plane error due to the Poisson effect for the specific application to be at the level of $0.4 \%$, a value that can be considered acceptable. We note that knowing the distance of the image plane to the specimen and other intrinsic camera parameters enables the correction for this error in an iterative manner. A yet more reliable option, that would also reduce any other out of plane movement error, would be to use a 3D full field measurement system utilizing a pair of cameras for achieving stereo-vision capability.

\section{Characterization Methodology}

The coordinate system definition is presented in Fig. 5. Neither the exact properties of the fiber woven roving fabric nor the properties of the epoxy were available. Consequently, the material is treated as a generally orthotropic symmetric material that can be described by the 9 independent engineering constants $\left\{E_{1}, E_{2}, E_{3}, v_{12}, v_{13}, v_{23}, G_{12}, G_{23}, G_{13}\right\}$.

\section{$<$ Figure 5 placed near hear $>$}

Furthermore, based on the geometrical symmetries of the material system presented in Fig. 5, it should be expected that the material exhibits square symmetry with six independent properties. In addition, in the absence of any specific information and because of possible misalignments from orthogonally introduced by the manufacturing process of the G-10 laminate, we assume that the following reductions may hold:

$$
\left.\begin{array}{l}
E_{1} \approx E_{2} \\
v_{13} \approx v_{23} \\
G_{13} \approx G_{23}
\end{array}\right\}
$$


Due to the absence of an estimate for the through the thickness shear moduli, the following expression can be used as a first order estimate as it relates to anisotropic fibrous composites [18]:

$$
G_{23} \approx G_{13} \approx \frac{E_{2}}{2\left(1+v_{23}\right)}
$$

For each of the experiments, stress-strain and strain-strain curves were identified and the tangent properties of Young's moduli and associated Poisson ratios were computed respectively. For each property pair of interest, the tangent properties calculation was performed using a least squares approximation of a quadratic curve of the form:

$$
\begin{aligned}
& f(x)=a x^{2}+b x+c \\
& f(x)=y
\end{aligned}
$$

In certain cases (like the tension experiments) where both stress and strain are tared to 0 , the coefficient $c$ in Eqn. (8) can be eliminated a priori. In any case, it is easy to show that the respective tangent modulus at the point where the free variable (e.g. stress) vanishes is:

$$
T=\left.\frac{d f(x)}{d x}\right|_{x \rightarrow 0}=b
$$

It should be noted that, in cases where not the entire range of the experimental specimen behavior curves was adequately approximated by a quadratic curve, only the initial portion of this curve was considered in the calculation. Additionally, this approach of identifying the tangent modulus has the advantage that it naturally tares the initial offset, if any, by encoding it in the parameter $c$ of Eqn. (8).

Some bending induced rotation of the specimen and the compression platens due to the small eccentricity of the specimen from the loading axis resulted in spurious strain values during the initial loading. It was observed that the load needed to be above $5 \mathrm{kN}$ for the friction to be sufficient to hold the specimen in place. In these cases, the original low-load portion of the experimental data was disregarded and the strain values were tared based on the inversion of the least squares approximation of Eqn. (8).

\subsection{Young's Moduli}

The tangent Young's moduli $\left(E_{1}, E_{2}, E_{3}\right)$ were calculated performing tension and compression experiments in the respective directions. In this case, the least squares approximation was performed by substituting $x=\varepsilon_{v}$ and $y=\sigma_{\infty}$ in Eqn. (8). $\sigma_{\infty}$ is the tensile or compressive stress experienced by the specimen and $\varepsilon_{v}$ is the strain along the loading axis, as calculated by DSI at the point indicated by the cross in Figs. $3 \mathrm{a}$ and $3 \mathrm{~b}$.

\subsection{Poisson's Ratios}

The tangent Poisson ratios $\left(v_{12}, v_{21}, v_{13}, v_{31}, v_{23}, v_{32}\right)$ were calculated by substituting $x=\varepsilon_{h}$ and $y=\varepsilon_{v}$ in Eqn. (8), where $\varepsilon_{v}$ is the strain component parallel to the loading direction and $\varepsilon_{h}$ 
is the strain component perpendicular to the loading direction, as calculated by DSI at the point indicated by the cross in Figs. 3a and 3b.

\subsection{Shear Modulus}

The in-plane tangent shear modulus $G_{12}$ was computed by performing tension experiments at $45^{\circ}$ relative to the orthotropic axes. The expression for the $G_{12}$ calculation can be derived by calculating the compliance matrix at this angle. For brevity, we performed the calculation using the in-plane components only. The compliance matrix at $0^{\circ}$ is:

$$
S=\left(\begin{array}{ccc}
\frac{1}{E_{1}} & -\frac{v_{12}}{E_{1}} & 0 \\
-\frac{v_{12}}{E_{1}} & \frac{1}{E_{1}} & 0 \\
0 & 0 & \frac{1}{G_{12}}
\end{array}\right)
$$

In order to perform the $45^{\circ}$ transformation of the compliance matrix, it must be first made compatible with the Hooke's law for anisotropic materials such that the tensorial shear strain $\varepsilon_{12}$ is half the engineering shear strain $\gamma_{12}$. This is achieved by the following the sequence of operations:

$$
S_{i j}^{e}=\left\{\begin{array}{l}
S_{i j}, i \neq 3 \\
\frac{1}{2} S_{i j}, i=3
\end{array}\right.
$$

Then, the transformed compliance matrix can be calculated by:

$$
S_{45}^{e}=T_{45}^{-1} S^{e} T_{45}
$$

where $T_{45}$ is a transformation matrix given by [18]:

$$
T_{45}=\left(\begin{array}{ccc}
m^{2} & n^{2} & 2 m n \\
n^{2} & m^{2} & -2 m n \\
-m n & m n & m^{2}-n^{2}
\end{array}\right)
$$

with $m=\cos 45$ and $n=\sin 45$. Finally,

$$
S_{45 i j}=\left\{\begin{array}{l}
S_{45 i j}^{e}, i \neq 3 \\
2 S_{45 i j}^{e}, i=3
\end{array}\right.
$$

In simple tension, the strain tensor will be (using Voigt notation): 


$$
\left(\begin{array}{c}
\varepsilon_{x x} \\
\varepsilon_{y y} \\
\varepsilon_{x y}
\end{array}\right)=S_{45}\left(\begin{array}{c}
0 \\
\sigma_{y y} \\
0
\end{array}\right)
$$

By considering only the expression for $\varepsilon_{y y}$ in Eqn. (15) and solving for $G_{12}$, we obtain:

$$
G_{12}=\frac{E_{1} E_{2} E_{45}}{4 E_{1} E_{2}-E_{1} E_{45}+2 E_{2} E_{45} \nu_{12}-E_{2} E_{45}}
$$

where $E_{45}$ is the apparent Young's modulus at a $45^{\circ}$ cut specimen and is calculated as described in Section 4.1. Neglecting variable correlations, we can calculate the propagation of uncertainty in Eqn. (16):

$$
\begin{aligned}
s_{G_{12}}^{2} & =\left(\frac{\partial G_{12}}{\partial E_{1}}\right)^{2} s_{E_{1}}^{2}+\left(\frac{\partial G_{12}}{\partial E_{2}}\right)^{2} s_{E_{2}}^{2}+ \\
& +\left(\frac{\partial G_{12}}{\partial E_{45}}\right)^{2} s_{E_{45}}^{2}+\left(\frac{\partial G_{12}}{\partial v_{12}}\right)^{2} s_{v_{12}}^{2}
\end{aligned}
$$

where $S_{v}$ is the standard deviation of the variable $v$. Performing the calculations yields:

$$
s_{G_{12}}=\sqrt{\frac{E_{45}^{4}\left(E_{1}^{4} s_{E_{2}}^{2}+E_{2}^{4}\left(\left(1-2 v_{12}\right)^{2} s_{E_{1}}^{2}+4 E_{1} s_{v_{12}}^{2}\right)\right)}{\left(E_{1}\left(E_{45}-4 E_{2}\right)+E_{2} E_{45}\left(1-2 v_{12}\right)\right)^{4}}}
$$

\subsection{Compression Tests}

The compression tests can be used to calculate additional components of the elastic stiffness or compliance tensor because specimens can be easily cut with different orientations. Unfortunately, because in general we shouldn't expect the same tension/compression properties, these results should be used only as an approximate value when considered in modeling tension effects.

For an orthotropic material, the following relationships hold [18] and the respective compliance tensor components are defined by:

$$
\begin{aligned}
& S_{12}=S_{21}=-\frac{v_{12}}{E_{1}}=-\frac{v_{21}}{E_{2}} \\
& S_{13}=S_{31}=-\frac{v_{13}}{E_{1}}=-\frac{v_{31}}{E_{3}} \\
& S_{23}=S_{32}=-\frac{v_{23}}{E_{2}}=-\frac{v_{32}}{E_{3}}
\end{aligned}
$$


Because of the experimental errors, the equality in Eq. (19) is not absolutely realizable. Hence, a homogenization process was employed to increase the certainty of the calculated result. For each of the equalities in (19) we consider the compliance tensor components as the average of the ones calculated from the compression experiments as:

$$
S_{i j}^{h}=S_{j i}^{h}=\frac{1}{2}\left(-\frac{v_{i j}}{E_{i}}-\frac{v_{j i}}{E_{j}}\right), i \neq j
$$

For the values of $S_{i j}^{h}$ s calculated in Eqns. (20) we calculated the $v_{i j}$ s using Eqns. (19).

\section{Results}

The experimental results are presented in Figs. 6 through 14. The orientation of the specimen with respect to the sheet stock is also shown on each individual figure. For the tension experiments, it can be observed that the experiments exhibited remarkable repeatability and, in many cases, the curves from individual tests are indistinguishable from each other.

However, the compression tests did not exhibit the same repeatability, but still they remained satisfactory. In this case, we did not load the specimens to failure as we were interested only in identifying the elastic properties. Because of the aberrations introduced by the experimental setup and the associated process (including specimen placement misalignment, platen-specimen tribological conditions, platen-grip alignment, system compliance, etc.), the initial part of the compression tests is deemed unreliable, as shown by the gray dashed lines in Figs. 9 through 14, and thus is discarded for the computations. For this reason, these data were not used in the calculation of the material properties.

$<$ Figures 6-14 placed near or after here $>$

The tensile tangent engineering properties of the material were identified to be (deviation indicate $95 \%$ confidence):

$$
\begin{array}{ll}
E_{1} & =24.63 \mathrm{GPa} \pm 0.44 \mathrm{GPa} \\
E_{2} & =27.38 \mathrm{GPa} \pm 1.71 \mathrm{GPa} \\
G_{12} & =5.52 \mathrm{GPa} \pm 0.11 \mathrm{GPa} \\
v_{12} & =0.194 \pm 0.032
\end{array}
$$

For the tensile experiments, the maximum stress reached for each orientation, along with the strain tensor components at those stress levels are presented in Table 1.

$<$ Table 1 placed near here $>$

The properties as calculated by the compression experiment data are presented in Eqn. (22), with deviations indicating $95 \%$ confidence: 


$$
\begin{aligned}
& E_{1}=27.392 \mathrm{GPa} \pm 3.53 \mathrm{GPa} \\
& E_{2}=30.674 \mathrm{GPa} \pm 2.78 \mathrm{GPa} \\
& E_{3}=11.493 \mathrm{GPa} \pm 0.56 \mathrm{GPa} \\
& v_{12}=0.249 \pm 0.0395 \\
& v_{13}=0.455 \pm 0.0490 \\
& v_{23}=0.518 \pm 0.0384
\end{aligned}
$$

Using Eqn. (7) we calculated:

$$
G_{23}=12.18 \mathrm{GPa} \pm 1.12 \mathrm{GPa}
$$

In an attempt to identify a single value for the material properties that can be used both in tension and compression, we used arguments of availability and of uncertainty. Hence, because of the low uncertainty of the results of the tension experiments and the higher uncertainty of the compression tests, we assumed the values of $E_{1}$ and $E_{2}$ and $v_{12}$ to be equal to those of the tension experiments. Because $G_{12}$ was calculated only for the tension case, we assumed its value to be the one calculated from the tension experiments. The rest of the properties $\left(E_{3}\right.$, $\left.G_{13} \approx G_{23}, V_{13}, v_{23}\right)$ were assumed to be those derived from the compression tests. The final homogenized properties based on these arguments are presented in Table 2. Finally, the compliance tensor in its matrix form as it is derived from the engineering properties [18] is shown in Eqn. (24).

$\boldsymbol{S}=\left(\begin{array}{cccccc}0.0406009 & -0.00787657 & -0.0184734 & 0 & 0 & 0 \\ -0.00787657 & 0.036523 & -0.0189189 & 0 & 0 & 0 \\ -0.0184734 & -0.0189189 & 0.0870095 & 0 & 0 & 0 \\ 0 & 0 & 0 & 0.0821018 & 0 & 0 \\ 0 & 0 & 0 & 0 & 0.0821018 & 0 \\ 0 & 0 & 0 & 0 & 0 & 0.181159\end{array}\right) \mathrm{GPa}^{-1}(24)$

For convenience the corresponding inverse of this matrix defines the Hooke's tensor components as follows,

$$
C=\left(\begin{array}{cccccc}
30.9462 & 11.3564 & 9.03961 & 0 . & 0 . & 0 . \\
11.3564 & 35.0228 & 10.0263 & 0 . & 0 . & 0 . \\
9.03961 & 10.0263 & 15.5923 & 0 . & 0 . & 0 . \\
0 . & 0 . & 0 . & 12.18 & 0 . & 0 . \\
0 . & 0 . & 0 . & 0 . & 12.18 & 0 . \\
0 . & 0 . & 0 . & 0 . & 0 . & 5.52
\end{array}\right) \mathrm{GPa}
$$




\section{Conclusions}

In this work we present results from the experimental testing of the G-10 composite material system under tensile and compressive mechanical loading. The tests were performed on specimens cut from sheet stock in various orientations in order to identify their anisotropic elastic material properties along with the associated confidence bounds. From these results, we have calculated the complete set of parameters necessary to characterize the G-10 material. These parameters are sufficient to conduct rigorous computational studies, such as finite-element analysis or other structural mechanical simulations, of structures and assemblies utilizing G-10. We anticipate that these results will be useful in the design of electromechanical systems such as electromagnetic launchers and high-power superconducting magnets.

In this work, the relatively new DSI methodology for full-field optical strain measurements was employed. The results shown in Figs. 6-14 demonstrate very good repeatability and, in the tensile tests, it is difficult to distinguish the curves associated with replicate specimens. This indicates that the DSI methodology is capable of producing highquality experimental data in the field of polymer and composite material testing. This also validates the arguments made in Section 3, and shows that a single-camera 2D strain measurement approach may be successfully used to characterize certain materials. We note that the rigidity of the camera mounting system and minimization of any relative movement between the camera and specimen reference frames is critical to this repeatability.

The data collected during the compressive tests contains several irregularities. While the data are still usable for characterization, repeatability between the replicate specimens is not as good as in the case of the tensile tests. In particular, it can be seen that the stress-strain curves between replicate specimens are offset. We attribute these irregularities primarily to misalignment of the specimens when placed between the platens; the specimens were aligned by hand using scribed marks on the platen surface. These misalignments may have been compounded by surface texture or tribological conditions at the specimen-platen interface. In Section 5, we present stress-strain curves for tests in each of the directions, tensile strength characteristics for three orientations and tangent properties as calculated by using these curves. One interesting observation derived from these experiments is that, although the material was expected to exhibit square symmetry based on the orthogonality of the fibers, it in fact follows a more general orthotropic behavior. This may be due to various causes but the most probable is the existence of a preferential direction developed from the manufacturing process. The testing of specimens produced by multiple manufacturers will be necessary to conclusively identify the presence and cause of general orthotropy in G-10 materials.

\section{Acknowledgments}

Funding for this project was provided by the Office of Naval Research (ONR) through the Naval Research Laboratory's (NRL) Basic Research Program. The authors also wish to thank Mr. Harry Jones of NRL Code 6350, for his advice and assistance carrying out the experiments detailed in this work, as well as Dr. Scott Douglass and Mr. Rich Cairns of NRL Code 6700, for supplying the raw G-10 material for the specimens.

\section{References}


[1] NEMA Standards Publication No. LI 1-1998, Industrial Laminating Thermosetting Products, National Electrical Manufacturers Association, Rosslyn, VA, 1998.

[2] IEC International Standard 60893-3-1:2012, Insulating materials - Industrial rigid laminated sheets based on thermosetting resins for electrical purposes - Part 3-1: Specifications for individual materials - Types of industrial rigid laminated sheets, International Electrotechnical Commision, Geneva, Switzerland, 2012.

[3] MIL-I-24768, Military Specification: Insulation, Plastics, Laminated, Thermosetting; General Specification for, United States Department of Defense, 1992.

[4] G. Lubin (ed.), Handbook of Composites, Van Nostrand Reinhold Company, New York, NY, 1982.

[5] S. Zherlitsyn, T. Herrmannsdorfer, Y. Skourski, A. Sytcheva, and J. Wosnitza, Design of Non-destructive Pulsed Magnets at the HLD, Journal of Low Temperature Physics, 146(516):719-732.

[6] S. Zherlitsyn, A.D. Bianchi, T. Herrmannsdoerfer, F. Pobell, Y. Skourski, A. Sytcheva, S. Zvyagin, and J. Wosnitza, Coil Design for Non-Destructive Pulsed-Field Magnets Targeting 100 T, IEEE Trans. on Applied Superconductivity, 16(2)1660-1663, 2006.

[7] P.A. Alekseev, A.I. Boev, V.E. Keilin, I.A. Kovalev, S.L. Kruglov, V.N. Lazukov, and I.P. Sadikov, Experimental evidence of considerable stability increase in superconducting windings with extremely high specific heat substances, Cryogenics, 44(11)763-766.

[8] T. Watt and M. Crawford, Experimental Results From a Two-Turn 40-mm Railgun, IEEE Trans. on Magnetics, 45(1)490-494, 2009.

[9] A. Challita, B.L. Maas, D. Bauer, and M. Heyse, A multiple armature railgun launcher, IEEE Trans. on Magnetics, 29(1)763-768, 1993.

[10] K. Ravi-Chandar and S. Satapathy. Mechanical Properties of G-10 Glass-Epoxy Composite. Technical Report, IAT.R 0466, Institute for Advanced Technology The University of Texas at Austin, 3925 W. Braker Lane, Suite 400, Austin, TX 78759-5316, 2007.

[11] Mechanical, Electrical, and Thermal Characterization of G-10CR and G-11CR GlassCloth/Epoxy Laminates Between Room Temperature and 4K, Advances in Cryogenic Engineering Materials, 26:235-244, 1980.

[12] Y. Shindo, R. Wang, and K. Horiguchi, Analytical and Experimental Studies of ShortBeam Interlaminar Shear Strength of G-10CR Glass-Cloth/Epoxy Laminates at Cryogenic Temperatures, J. Eng. Mater. Technol, 123(1):112-118, 2000.

[13] A. Dasgupta, K.K. Agarwal, and S.M. Bhandarkar, Three-Dimensional Modeling of 
Woven-Fabric Composites for Effective Thermo-Mechanical and Thermal Properties, Composites Science and Technology, 56:209-223, 1996.

[14] L. Lamberson, Investigations of High Performance Fiberglass Impact Using a Combustionless Two-stage Light-gas Gun, Procedia Engineering 103:341-348, 2015.

[15] N. P. Andrianopoulos, Full-Field Displacement Measurement of a Speckle Grid by using a Mesh-Free Deformation Function. Strain, 42(4):265-271, 2006.

[16] N. P. Andrianopoulos, and A. P. Iliopoulos, Strain measurements by a hybrid experimental-numerical method using a mesh-free field function. Honorary Volume for Professor PS Theocaris, pages 31-41. Armenian Academy of Sciences, 2005.

[17] N.P. Andrianopoulos and A.P. Iliopoulos, Displacements Measurement in Irregularly Bounded Plates Using Mesh Free Methods. In Gdoutos, E.E., editors, Fracture of Nano and Engineering Materials and Structures, pages 587-588. Springer Netherlands, 2006.

[18] I.M. Daniel and O. Ishai. Engineering Mechanics of Composite Materials. Oxford University Press, USA, 2nd edition, 2005.

[19] A. Iliopoulos and J. G. Michopoulos, Effects of Anisotropy on the Performance Sensitivity of the Mesh-Free Random Grid Method for Whole Field Strain Measurement. ASME Conference Proceedings, 2009(48999):65-74, 2009.

[20] A.P. Iliopoulos, Digital Image Processing on Experimental Mechanics and Applications on Metallic Sheets. PhD thesis, National Technical University of Athens, 2007.

[21] A.P. Iliopoulos and N.P. Andrianopoulos. An Approach to Analyse Errors Introduced in the Random Grid Strain Measurement Method. Strain, 46(3):258-266, 2008.

[22] A.P. Iliopoulos, J.G. Michopoulos, and N.P. Andrianopoulos, Performance Analysis of the Mesh-Free Random Grid Method for Full-Field Synthetic Strain Measurements. Strain, 48(1):1-15, 2012.

[23] A.P. Iliopoulos and J.G. Michopoulos and N. P. Andrianopoulos. Performance Sensitivity Analysis of the Mesh-Free Random Grid Method for Whole Field Strain Measurements. ASME Conference Proceedings, 2008(43277):545-555, 2008.

[24] A.P. Iliopoulos and J.G. Michopoulos, Meshless methods for full field displacement and strain measurement. In Advances in Computers and Information in Engineering Research, pages 97-124. ASME, New York, 2014.

[25] A.P. Iliopoulos, and J.G. Michopoulos, Direct strain tensor approximation for full-field strain measurement methods. International Journal for Numerical Methods in Engineering, 95(4):313-330, 2013. 
[26] A.P. Iliopoulos, J.G. Michopoulos, A.C. Orifici and R.S. Thomson, Experimental Validation of the 2D Meshless Random Grid Method. ASME Conference Proceedings, 2011(54792):513-520, 2011.

[27] J.G. Michopoulos and A.P. Iliopoulos. A Computational Workbench for Remote Full Field 2D Displacement and Strain Measurements, ASME Conference Proceedings, 2009(48999):55-63, 2009.

[28] J.G. Michopoulos and A.P. Iliopoulos. A Computational Workbench for Remote Full Field 3D Displacement and Strain Measurements, ASME Conference Proceedings, 2011(54792):489-498, 2011.

\section{Figure Captions}

Figure 1: Experimental setup notional sketch.

Figure 2: Photograph of experimental setup. The strain imaging camera is pointed at a tension specimen placed in the grips of the Instron servohydraulic test frame.

Figure 3: Illustration of compressive (left) and tension (right) test specimens. All dimeinsions in $\mathrm{mm}$. Dots depict markers for the DSI method and crosses indictes the strain evaluation point.

Figure 4: Section of a pinhole camera model depicting the effect of the point $x$ projection on the camera plane at point $\boldsymbol{x}^{\prime}$ in the absence of through thickness Poisson effect and of the point $\boldsymbol{y}$ projection on the camera plane at point $\boldsymbol{y}^{\prime}$ in the presence of through thickness Poisson effect.

Figure 5: Coordinate axes definition of the G-10 composite material system. The cross-hatch lines indicate the woven roving orientation.

Figure 6: G-10 Tension experiment stress-strain curves with direction 2-2 parallel with the load. The figure depicts three tests, two of which exhibited proper failure behavior. In a certain range the curves are hard to distinguish.

Figure 7: G-10 Tension experiment stress - strain curves for a $45^{\circ}$ orientation. $\varepsilon_{h 45}$ indicate the lateral to the load strain, $\varepsilon_{v 45}$ the loading direction strain and $\varepsilon_{s 45}$ the shear strain. The figure depicts the data from three tests, one of which exhibited proper failure behavior. In a certain range the curves are hard to distinguish.

Figure 8: G-10 Tension experiment stress - strain curves with direction 1-1 parallel with the load. The figure depicts three tests, all of which exhibited proper failure.

Figure 9: G-10 Compression experiment stress - strain curves for the through the thickness (3-3) orientation. Camera plane parallel to the 1-3 plane. Specimen shown from the perspective of the camera.

Figure 10: G-10 Compression experiment stress - strain curves for the through the thickness (33 ) orientation. Camera plane parallel to the 2-3 plane. Specimen shown from the perspective of the camera. 
Figure 11: G-10 Compression experiment stress - strain curves for the 2-2 orientation. Camera plane parallel to the 1-2 plane. Specimen shown from the perspective of the camera.

Figure 12: G-10 Compression experiment stress - strain curves for the 2-2 orientation. Camera plane parallel to the 3-2 plane. Specimen shown from the perspective of the camera.

Figure 13: G-10 Compression experiment stress - strain curves for the 1-1 orientation. Camera plane parallel to the 2-1 plane. Specimen shown from the perspective of the camera.

Figure 14: G-10 Compression experiment stress - strain curves for the 1-1 orientation. Camera plane parallel to the 3-1 plane. Specimen shown from the perspective of the camera.

\section{Tables}

Table 1: Strength characteristics. The reported strains correspond at the $\sigma_{\max }$ level of stress.

\begin{tabular}{|l|l|l|l|l|}
\hline $\begin{array}{l}\text { Loading } \\
\text { Direction } \\
(y y)\end{array}$ & $\sigma_{\max }(\mathrm{MPa})$ & $\varepsilon_{x x}(\%)$ & $\varepsilon_{y y}(\%)$ & $\varepsilon_{x y}(\%)$ \\
\hline 11 & $310.1 \pm 6.4$ & -0.2 & 1.86 & -0.06 \\
\hline $45^{\circ}$ & 191 & -13.71 & 15.1 & 0.53 \\
\hline 22 & 366 & -0.25 & 1.8 & -0.01 \\
\hline
\end{tabular}

Table 2: G-10 tangent engineering properties. Error values indicate 95\% confidence.

\begin{tabular}{|l|l|l|l|}
\hline Quantity & Value & Error & Units \\
\hline$E_{1}$ & 24.63 & \pm 0.44 & $\mathrm{GPa}$ \\
\hline$E_{2}$ & 27.38 & \pm 1.71 & $\mathrm{GPa}$ \\
\hline$E_{3}$ & 11.49 & \pm 0.56 & $\mathrm{GPa}$ \\
\hline$G_{12}$ & 5.52 & \pm 0.11 & $\mathrm{GPa}$ \\
\hline$G_{13}$ & 12.18 & \pm 1.12 & $\mathrm{GPa}$ \\
\hline$G_{23}$ & 12.18 & \pm 1.12 & $\mathrm{GPa}$ \\
\hline$v_{12}$ & 0.194 & \pm 0.0320 & \\
\hline$v_{13}$ & 0.455 & \pm 0.0490 & \\
\hline$v_{23}$ & 0.518 & \pm 0.0384 & \\
\hline
\end{tabular}


Fig. 1

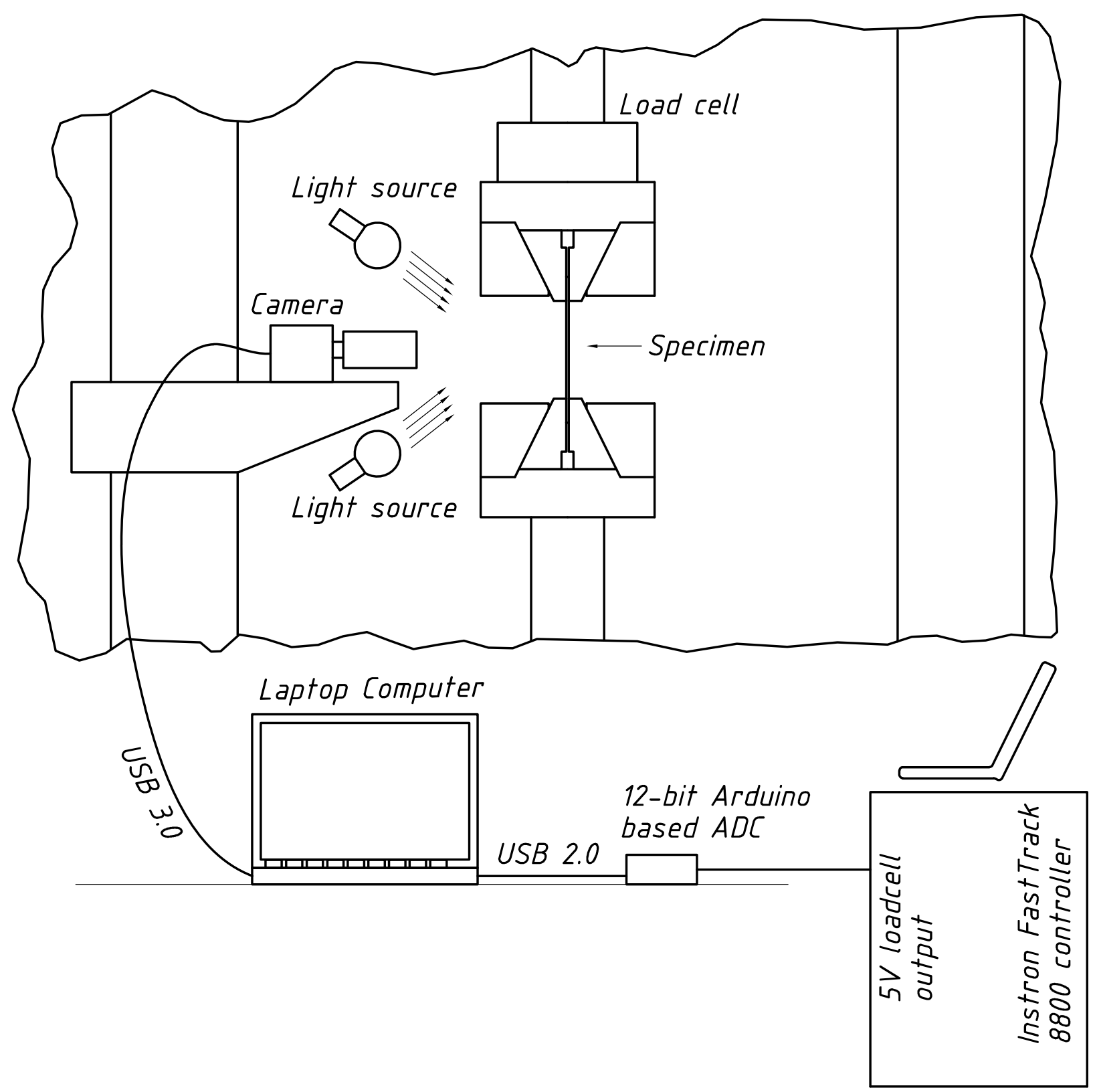


Fig. 2

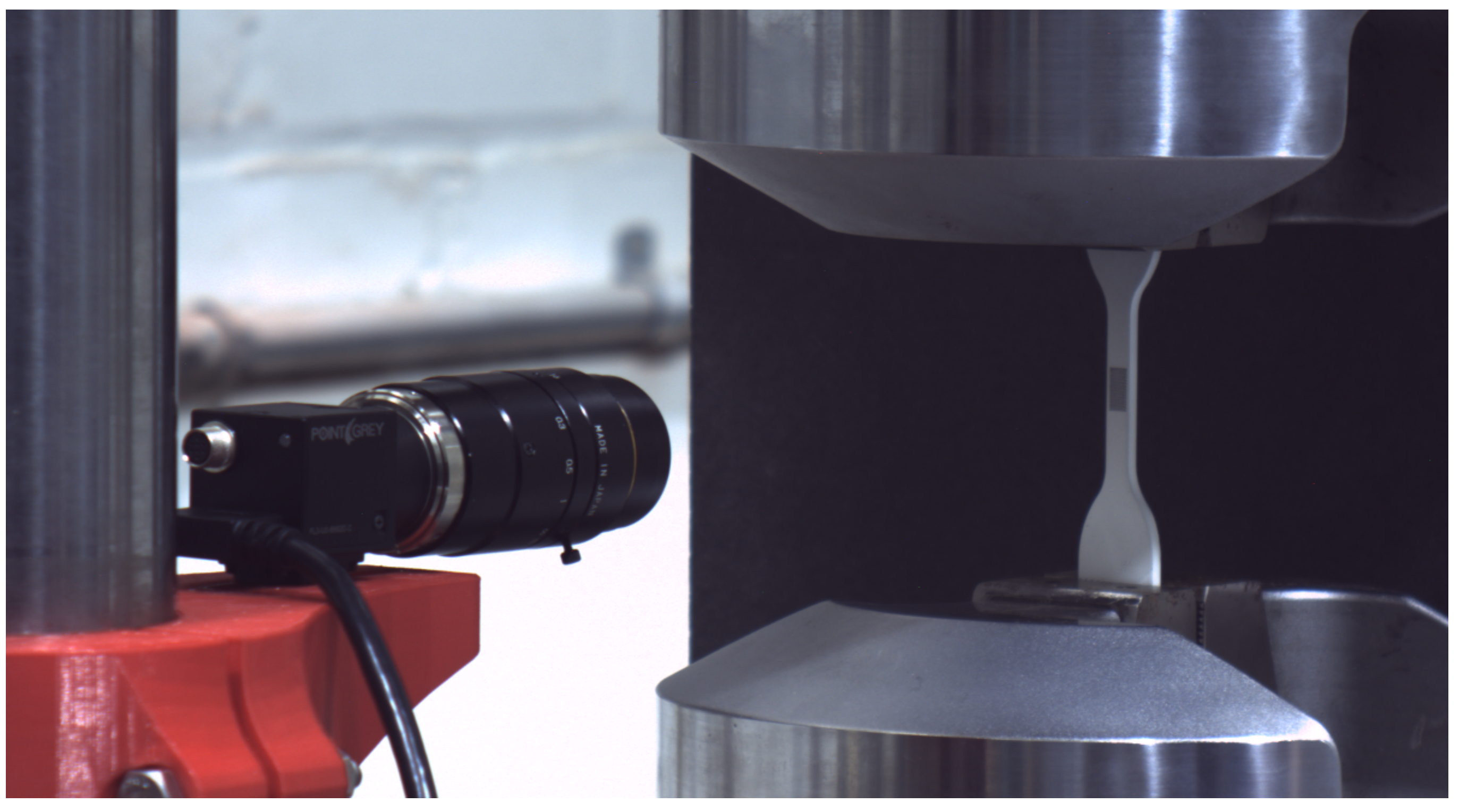


Fig. 3a
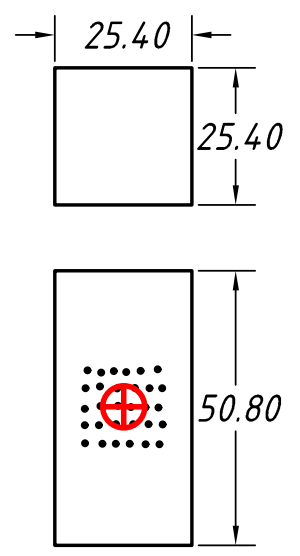

Fig. 3b

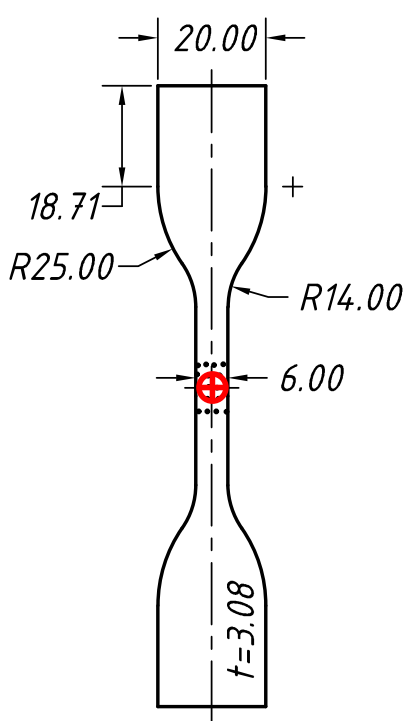


Fig. 4

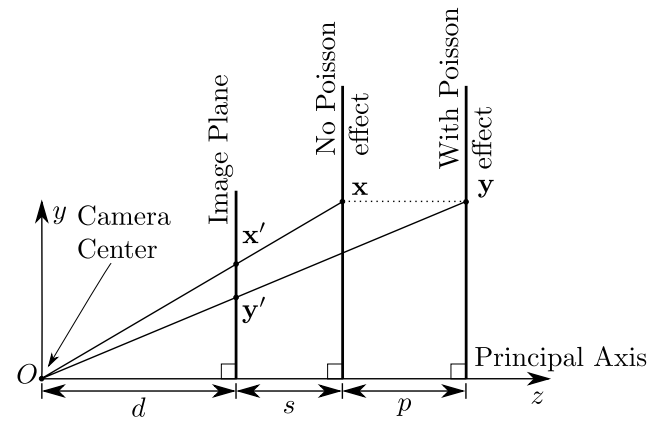


Fig. 5

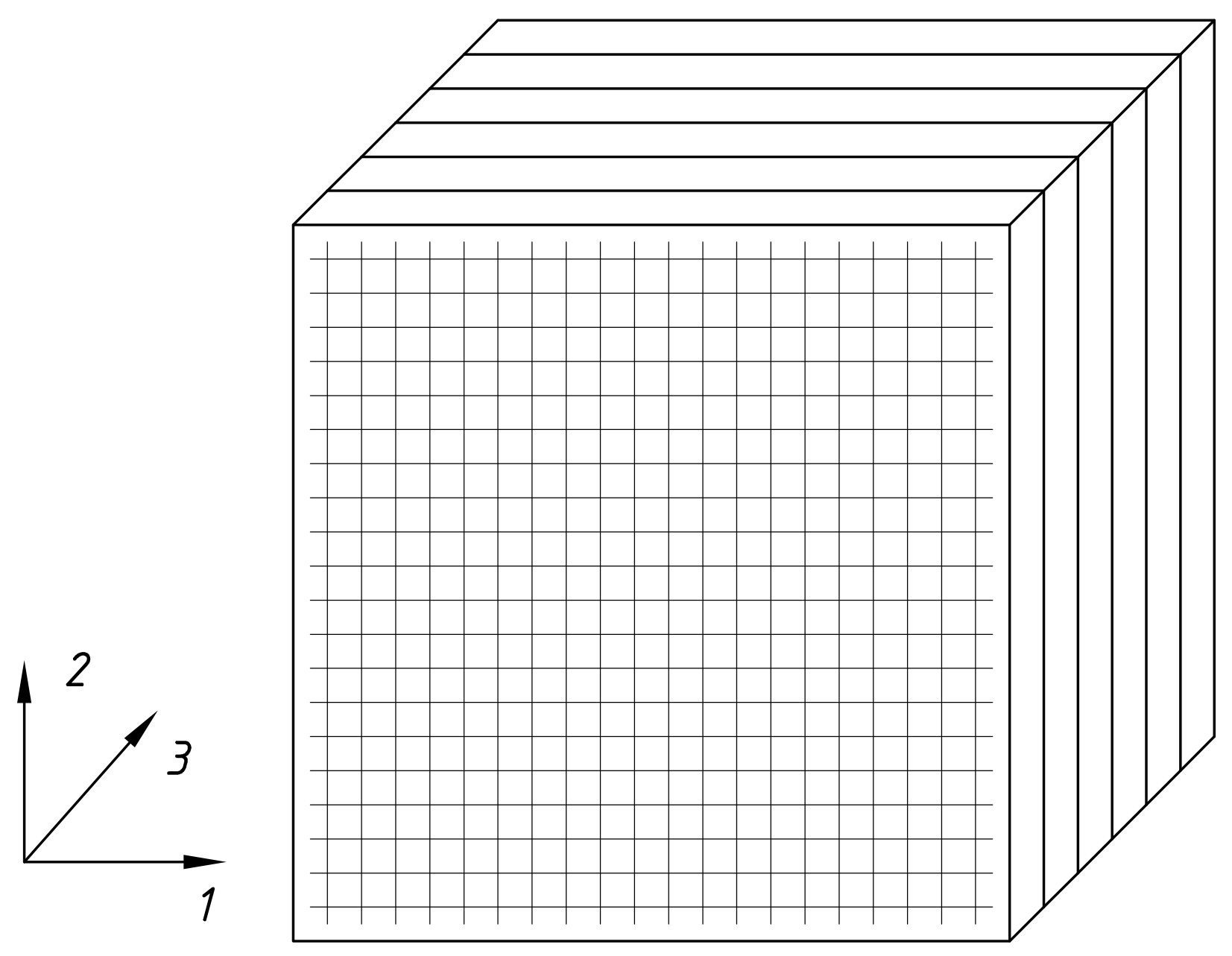


Fig. 6

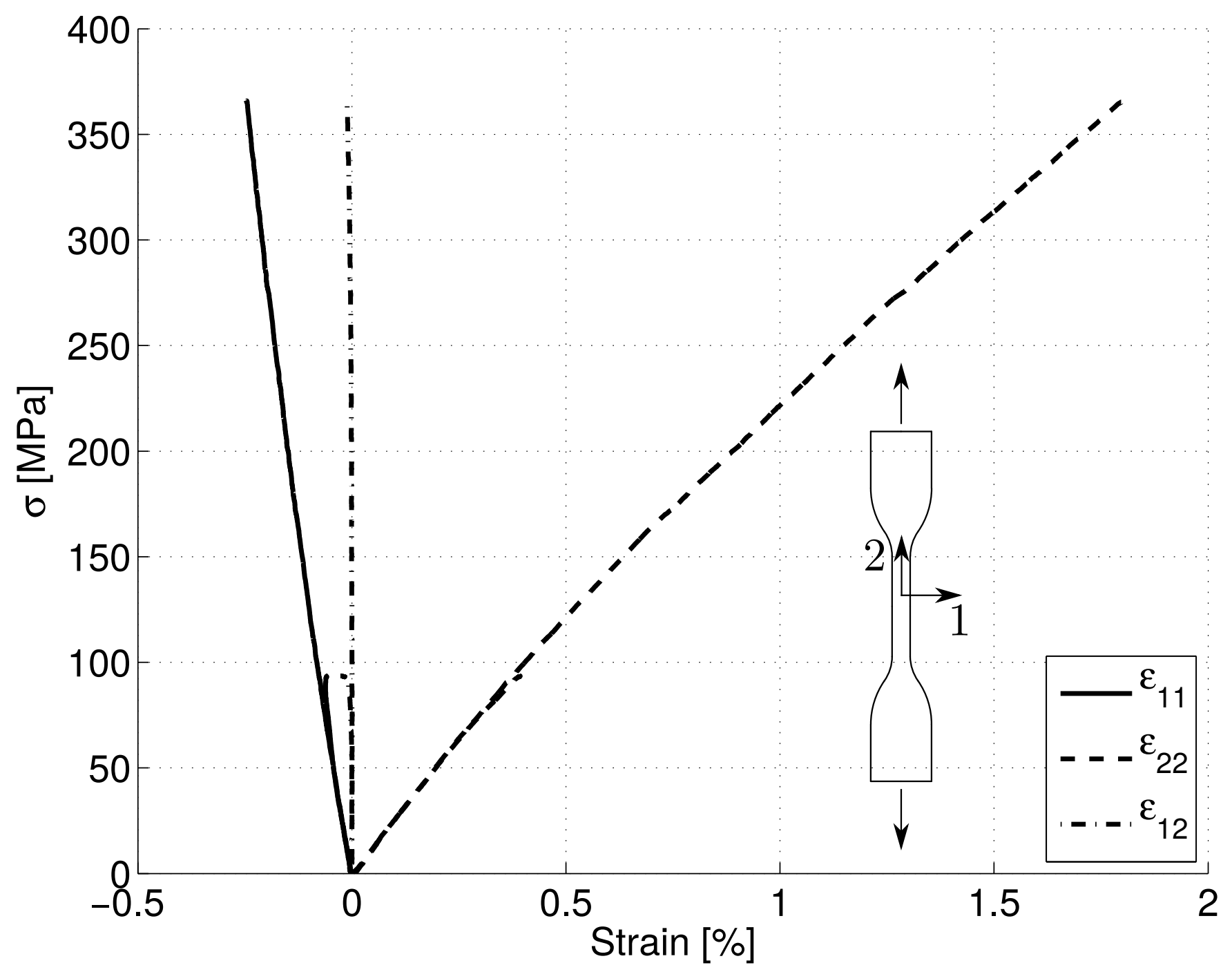


Fig. 7

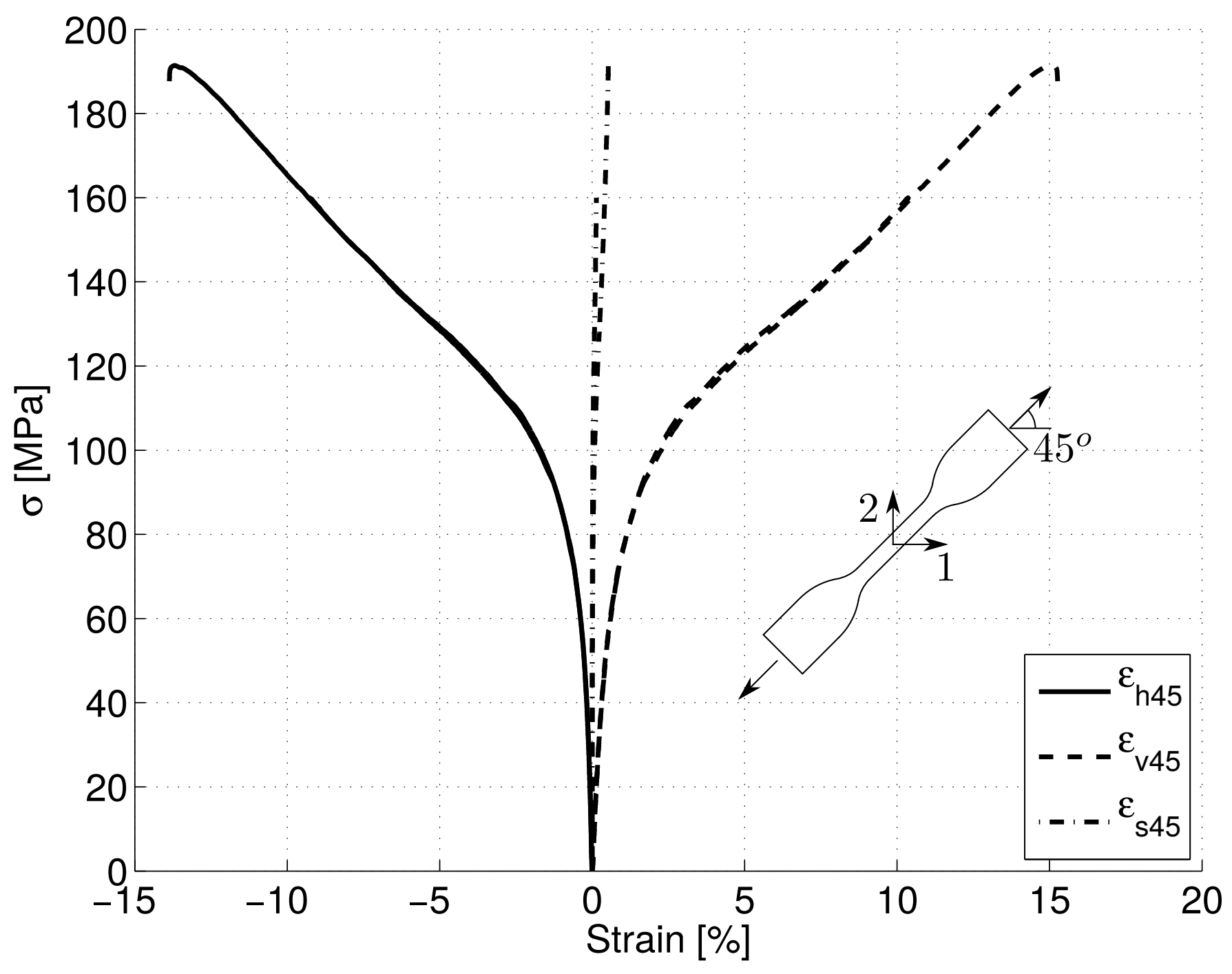


Fig. 8

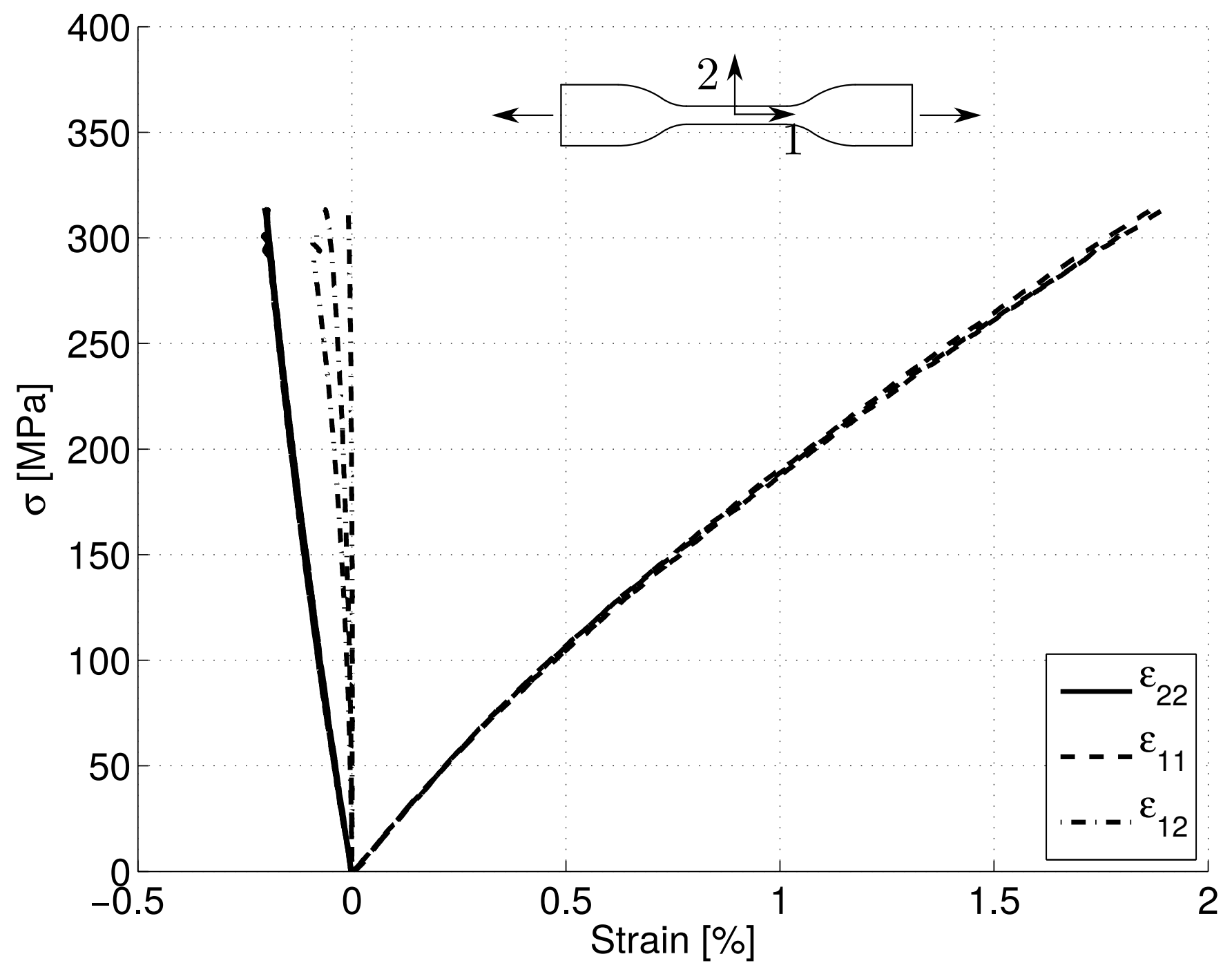


Fig. 9

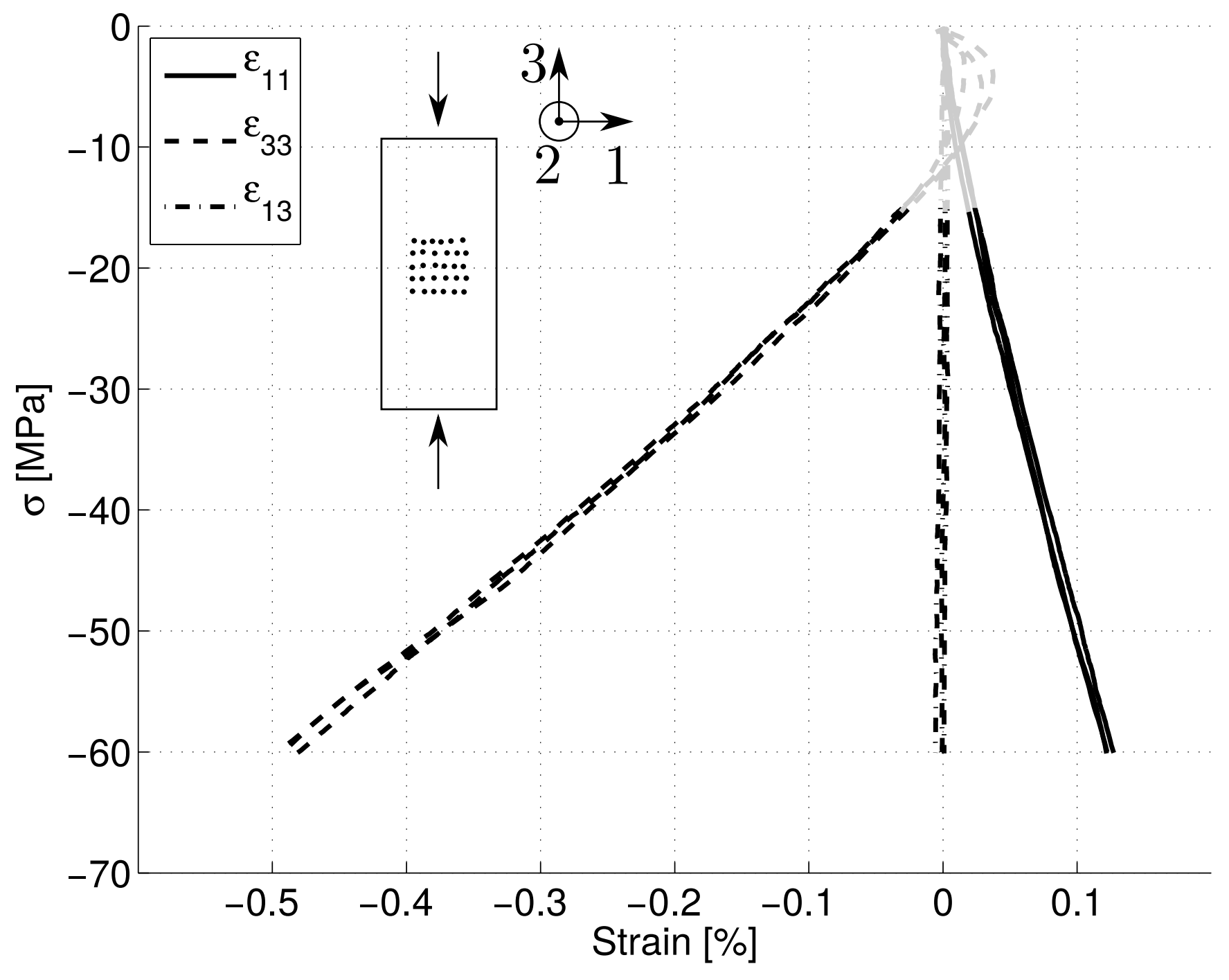


Fig. 10

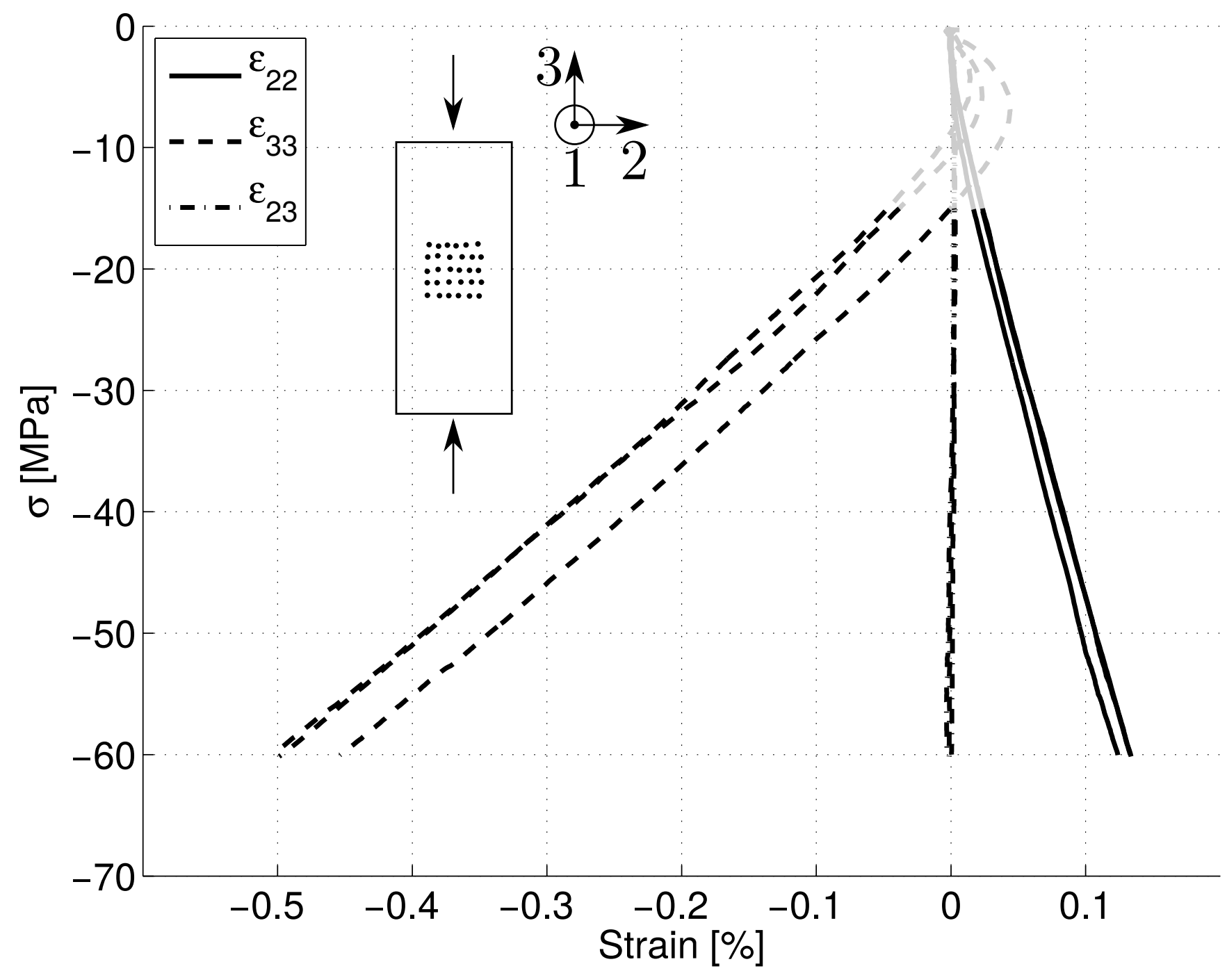


Fig. 11

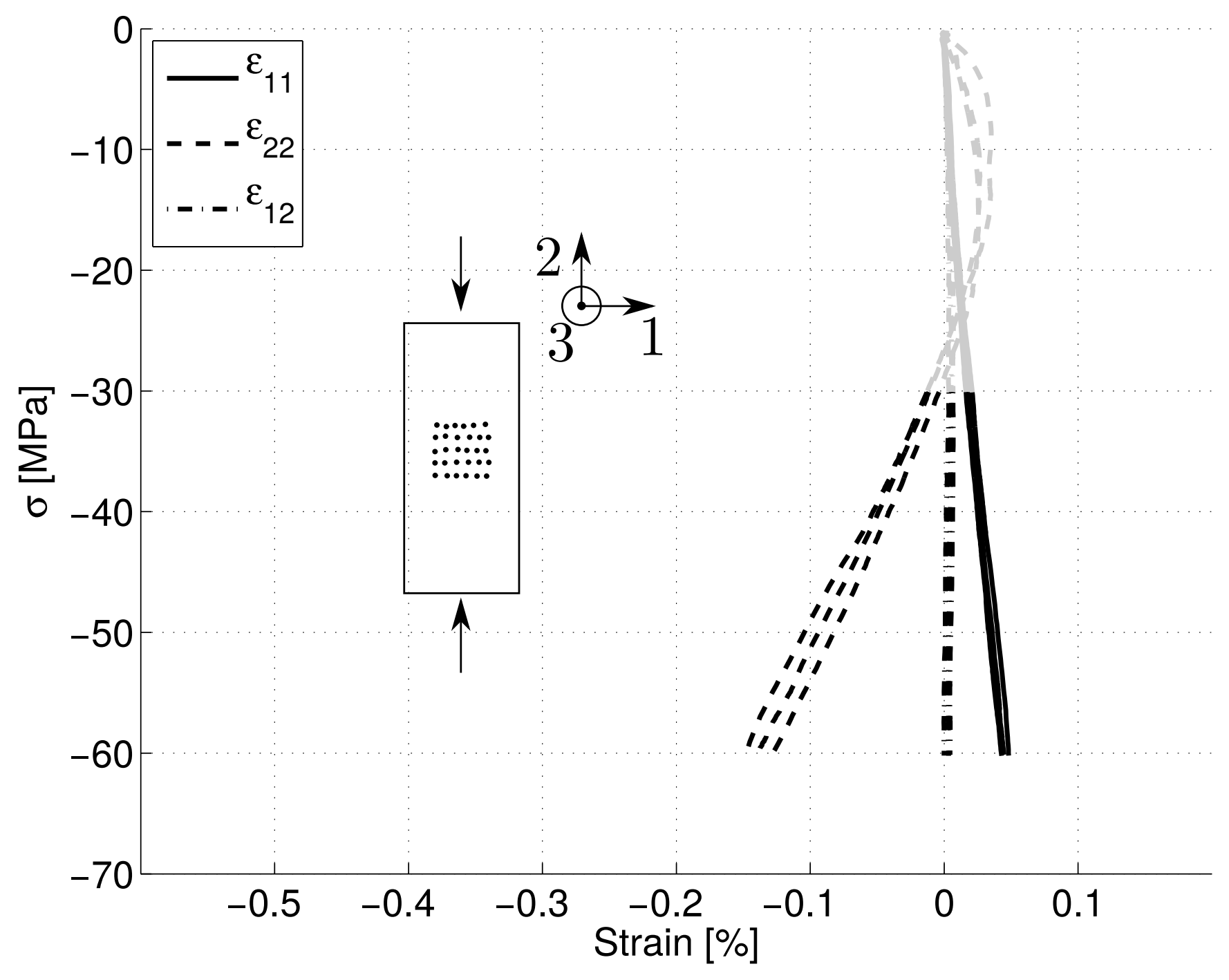


Fig. 12

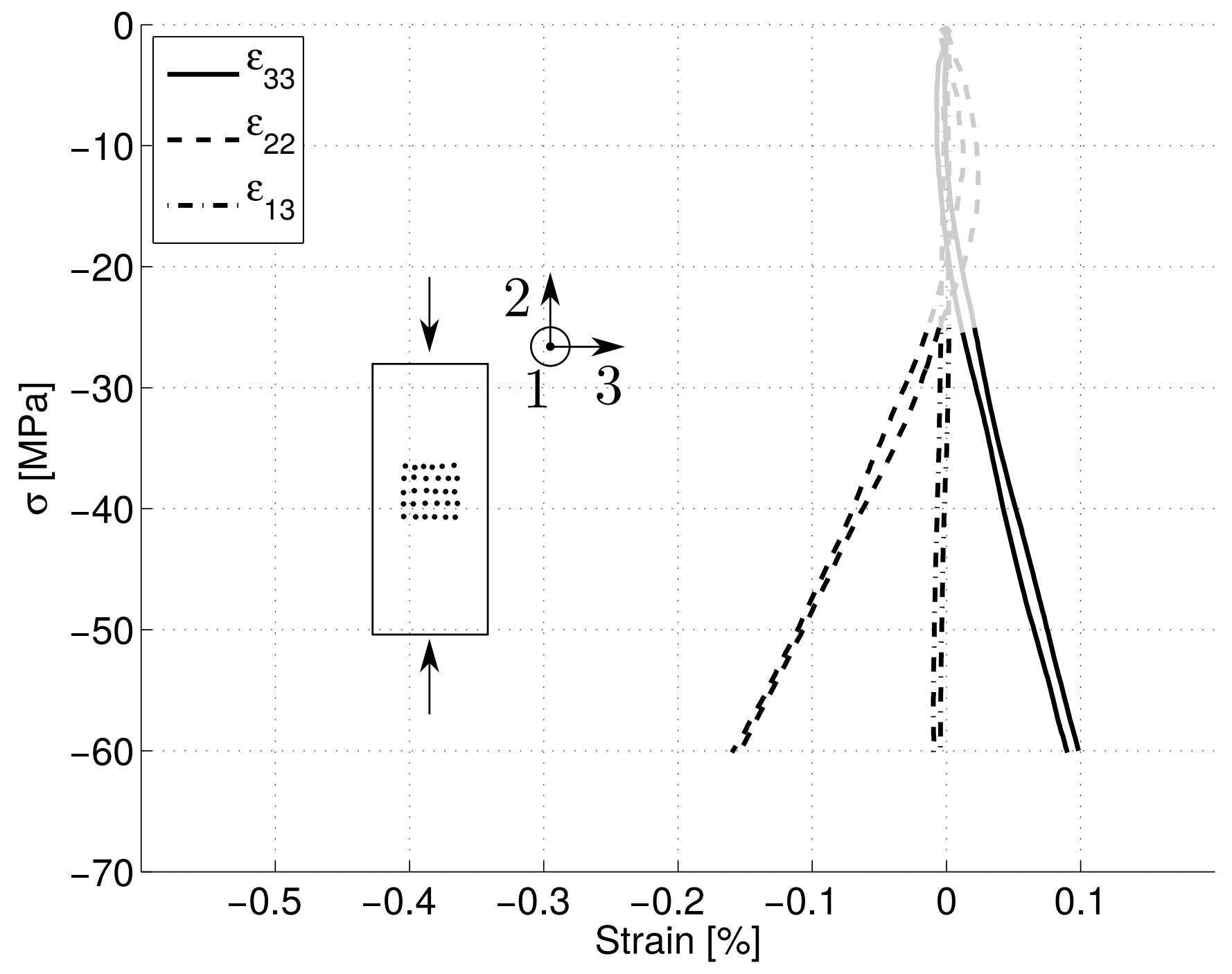


Fig. 13

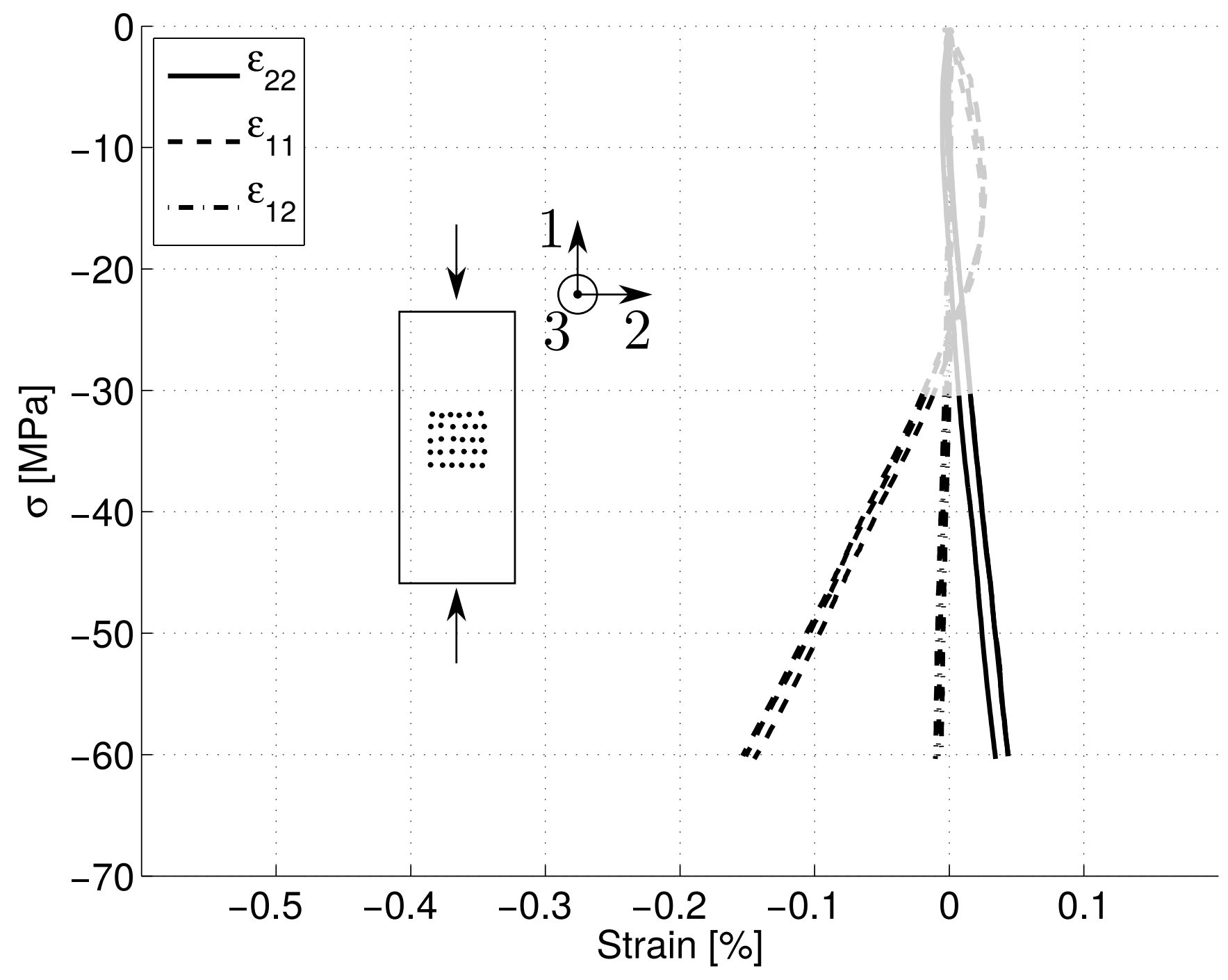


Fig. 14

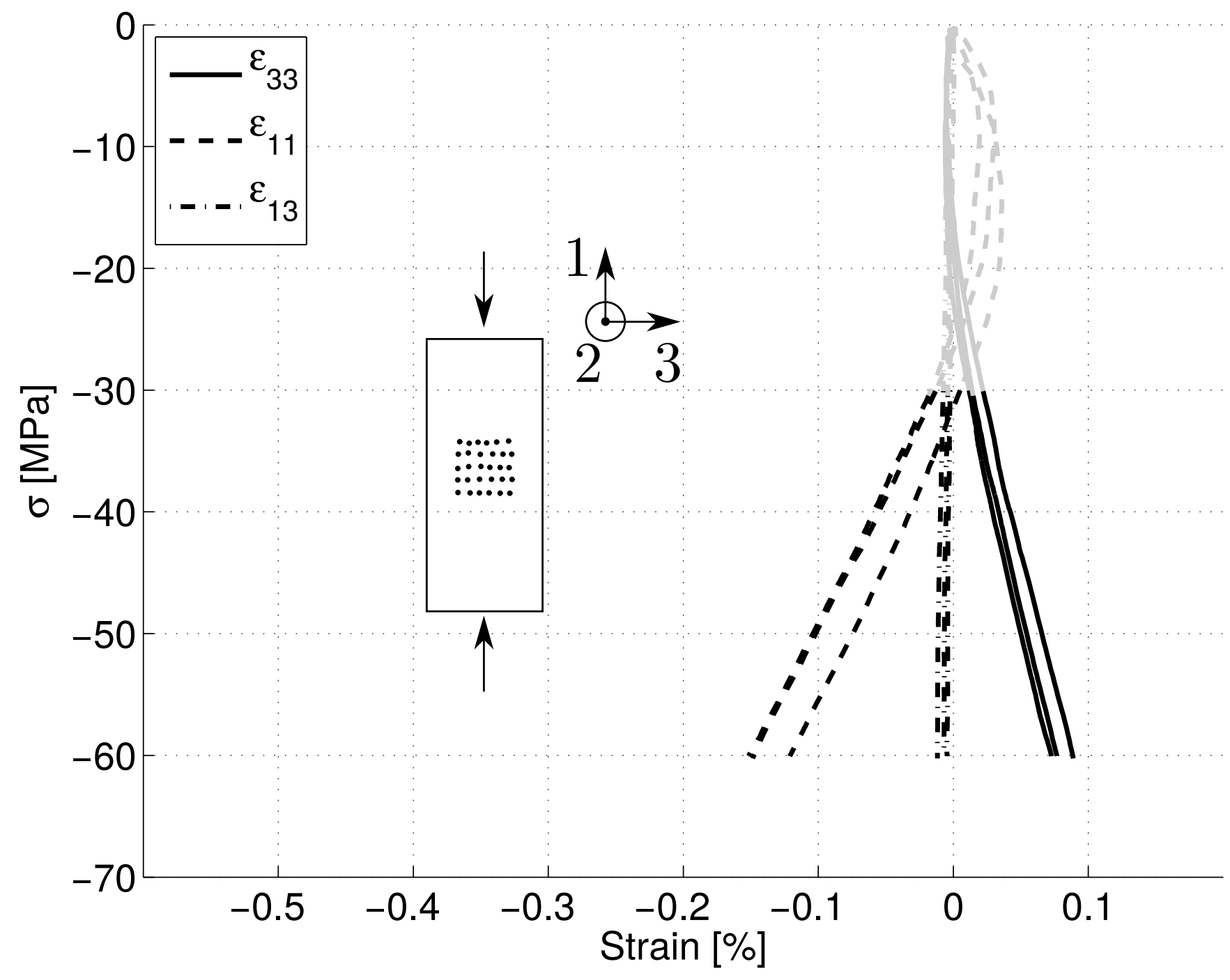

